hep-ph/9811252

PRL-TH-98/011

\title{
Vacuum Solutions of Neutrino Anomalies Through a Softly Broken $U(1)$ symmetry
}

\author{
Anjan S. Joshipura and Saurabh D. Rindani \\ Theoretical Physics Group, Physical Research Laboratory, \\ Navarangpura, Ahmedabad, 380 009, India.
}

\begin{abstract}
We discuss an extended $S U(2) \times U(1)$ model which naturally leads to mass scales and mixing angles relevant for understanding both the solar and atmospheric neutrino anomalies in terms of the vacuum oscillations of the three known neutrinos. The model uses a softly broken $L_{e}-L_{\mu}-L_{\tau}$ symmetry and contains a heavy scale $M_{H} \sim 10^{15} \mathrm{GeV}$. The $L_{e}-L_{\mu}-L_{\tau}$ symmetric neutrino masses solve the atmospheric neutrino anomaly while breaking of $L_{e}-L_{\mu}-L_{\tau}$ generates highly suppressed radiative mass scale $\Delta_{S} \sim 10^{-10} \mathrm{eV}^{2}$ needed for the vacuum solution of the solar neutrino problem. All the neutrino masses in the model are inversely related to $M_{H}$, thus providing seesaw-type of masses without invoking any heavy right-handed neutrinos. Possible embedding of the model into an $S U(5)$ grand unified theory is discussed.
\end{abstract}


Recent results on the oscillations of the muon neutrino seen at the $\mathrm{Su}-$ perkamioka [1] may be taken as the first experimental evidence for physics beyond the standard electroweak model. It is attractive to suppose that these are indirect hints into grand unification. The neutrino mass in the $S U(3) \times S U(2) \times U(1)$ theory can be characterized by a five dimensional operator which leads to $m_{\nu} \sim \frac{\langle\phi\rangle^{2}}{M},\langle\phi\rangle \sim 250 \mathrm{GeV}$ being the electroweak and the $M$ some heavy scale. The identification of $M$ with a scale $M_{H} \sim 10^{15} \mathrm{GeV}$ in grand unified theory nicely fits in [2, 3] with the neutrino mass scale $\sqrt{\Delta_{A}} \sim 0.07 \mathrm{eV}$ seen at the Superkamioka.

The seesaw model based on grand unified $S O(10)$ theory leads to the above dimension five term in which $M$ is determined by the right-handed neutrino masses. Apart from providing an overall scale, this model also relates [4] hierarchy among neutrino masses to that in the masses of the other (up quarks in the minimal case) fermions. This feature of $S O(10)$ can indeed provide another scale $\Delta_{S}$ needed to solve the solar neutrino problem. In the simplest $S O(10)$ model one expects $\frac{\Delta_{S}}{\Delta_{A}} \sim\left(\frac{m_{c}}{m_{t}}\right)^{4} . \Delta_{A} \sim 10^{-3} \mathrm{eV}^{2}$ then automatically leads to a $\Delta_{S}$ required for the vacuum solution [5] to the solar neutrino problem. The two large mixing angles needed in this case are not generic features of the seesaw model but could come out under reasonable assumptions. [6, 7].

The above attractive features of $S O(10)$ related to neutrino masses are not shared by generic $S U(5)$-based grand unified models. It is possible in these models to obtain neutrino masses and also to understand their overall scale in terms of the grand unified scale simply by adding a heavy 15 -dimensional Higgs field [2, 4, 8]. But one cannot easily relate hierarchy in $\Delta_{S}$ and $\Delta_{A}$ to the known fermion masses as in the $S O(10)$ case. Our aim here is to present a simple $S U(5)$ scheme which does this. While the mechanism we discuss is more general, we give a specific example in which (a) $\frac{\Delta_{S}}{\Delta_{A}}$ gets related to the charged lepton masses and (b) two large mixing angles come out naturally. The natural value for the $\frac{\Delta_{S}}{\Delta_{A}}$ is close to $10^{-7}$ resulting in vacuum oscillations as the cause for both the solar and atmospheric neutrino deficits.

To simplify the matter we shall first discuss a scheme based on the standard $S U(2) \times U(1)$ model and discuss its $S U(5)$ generalization later on. We need to extend $S U(2) \times U(1)$ model in two ways. We enlarge it with two extra multiplets of scalar fields namely a triplet $\Delta$ and an additional doublet field $\phi_{2}$. We also impose a global $L_{e}-L_{\mu}-L_{\tau}$ symmetry. This symmetry 
has been recognized [2, 6] to provide under reasonable assumptions two large mixing angles needed for the vacuum solutions of the neutrino anomalies. It leads to a pair of degenerate neutrinos with a common mass $m_{0}$ which determine the atmospheric neutrino mass scale. $m_{0}$ is inversely related to the grand unified scale $M_{H}$ in the manner discussed below.

Keeping $S U(5)$ unification in mind, we assume the triplet to be very heavy, with mass $\sim M_{H}$. But such a heavy triplet can influence the low energy theory crucially by generating $L_{e}-L_{\mu}-L_{\tau}$ symmetric neutrino mass matrix at tree level and departure from it at one-loop level.

The leptonic Yukawa couplings in the model are given by

$$
-\mathcal{L}_{Y}=\frac{1}{2} f_{i j} \bar{l}_{i L}^{c \prime} \Delta l_{j L}^{\prime}+\Gamma_{i j}^{a} \bar{l}_{i L}^{\prime} e_{j R}^{\prime} \phi^{a}+\text { H.c. },
$$

where $a=1,2$ label the Higgs doublets and $\Delta$ is $2 \times 2$ matrix in the $S U(2)$ space. The $L_{e}-L_{\mu}-L_{\tau}$ symmetry allows the following Yukawa textures:

$$
\begin{aligned}
& f \equiv \frac{M_{0}^{\nu}}{\left\langle\Delta^{0}\right\rangle}=\frac{m_{0}}{\left\langle\Delta^{0}\right\rangle}\left(\begin{array}{ccc}
0 & c & s \\
c & 0 & 0 \\
s & 0 & 0
\end{array}\right) ; \\
& \Gamma_{1} \equiv \frac{M_{1}^{l}}{\left\langle\phi_{1}^{0}\right\rangle}=\frac{1}{\left\langle\phi_{1}^{0}\right\rangle}\left(\begin{array}{ccc}
m_{1} & 0 & 0 \\
0 & m_{2} & m_{23} \\
0 & m_{32} & m_{3}
\end{array}\right) ; \\
& \Gamma_{2} \equiv \frac{M_{2}^{l}}{\left\langle\phi_{2}^{0}\right\rangle}=\frac{1}{\left\langle\phi_{2}^{0}\right\rangle}\left(\begin{array}{ccc}
0 & m_{12} & m_{13} \\
0 & 0 & 0 \\
0 & 0 & 0
\end{array}\right) ;
\end{aligned}
$$

where we have chosen the $L_{e}-L_{\mu}-L_{\tau}$ charge 2 for the field $\phi_{2}$ and zero for $\phi_{1}$ and $\Delta$.

The tree level neutrino mass matrix is $L_{e}-L_{\mu}-L_{\tau}$ symmetric and can be diagonalized by

$$
U^{\nu}=\left(\begin{array}{ccc}
1 / \sqrt{2} & -1 / \sqrt{2} & 0 \\
c / \sqrt{2} & c / \sqrt{2} & -s \\
s / \sqrt{2} & s / \sqrt{2} & c
\end{array}\right)
$$


If mixing among charged leptons is small then $U^{\nu}$ provides the bimaximal mixing [9] for $c \sim s \sim \frac{1}{\sqrt{2}}$ and can therefore simultaneously solve the solar and atmospheric neutrino anomaly through the vacuum oscillations.

The atmospheric scale $m_{0}$ is determined in the model by the vacuum expectation value (vev) of $\Delta^{0}$. This is driven by the following scalar potential

$$
\begin{aligned}
V & =M_{a}^{2} \phi_{a}^{\dagger} \phi_{a}+M_{H}^{2} \operatorname{Tr} . \Delta^{\dagger} \Delta \\
& +\lambda_{a}\left(\phi_{a}^{\dagger} \phi_{a}\right)^{2}+\lambda_{\Delta} \operatorname{Tr} .\left(\Delta^{\dagger} \Delta\right)^{2}+\ldots \\
& -\left[\mu_{a b} \phi_{a}^{T} \Delta \phi_{b}+c . c .\right] .
\end{aligned}
$$

The terms not explicitly written in the above equations correspond to some of the quartic terms involving $\Delta$ and quartic cross terms for the doublet fields. The trilinear terms in eq.(4) are of crucial importance. Firstly, they induce a small vev for the neutral Higgs $\Delta^{0}$ leading to a degenerate pair of neutrinos. In addition, they softly break the lepton number and $L_{e}-L_{\mu}-L_{\tau}$ symmetry. This breaking makes the model phenomenologically acceptable which otherwise would have contained a doublet plus triplet majoron already ruled out at LEP. In addition, the $L_{e}-L_{\mu}-L_{\tau}$ breaking by trilinear terms also generate radiative corrections to the neutrino mass matrix which result in the splitting of the degenerate pairs and solves the solar neutrino problem.

The triplet vev following from eq.(4) after minimization is of the order

$$
\left\langle\Delta^{0}\right\rangle \sim \frac{\left\langle\phi_{1}\right\rangle\left\langle\phi_{2}\right\rangle}{M_{H}}
$$

where $\mu_{a b}$ are assumed to be of the same order as the (large) triplet mass $M_{H}$. The neutrino mass generated at tree level thus displays the seesaw type dependence on the heavy scale. Specifically, one gets through eq.(2) $m_{0} \sim 3\left(10^{-1}-10^{-2}\right) \mathrm{eV}$ for $m_{H} \sim 10^{14}-10^{15} \mathrm{GeV}$ and $\left\langle\phi_{1}\right\rangle \sim\left\langle\phi_{2}\right\rangle$ providing the atmospheric neutrino scale.

The tree level neutrino mass matrix following from eq.(汭) is $L_{e}-L_{\mu}-L_{\tau}$ symmetric but the presence of a vev for $\phi_{2}$ breaks this symmetry in the charged lepton mass matrix. This breaking ultimately gets communicated to the neutrino mass matrix at the one-loop level. This occurs through the one loop diagrams shown in Fig.(1).

Let us define the charge lepton mass eigenstates as $e_{i L, R} \equiv U_{i \alpha}^{\dagger L, R} e_{\alpha L, R}^{\prime}$ where

$$
U^{L \dagger}\left(M_{1}^{l}+M_{2}^{l}\right) U^{R} \equiv U^{L \dagger} M^{l} U^{R}=M_{0}^{l}
$$


$M_{0}^{l}$ being the diagonal charged lepton mass matrix. The soft breaking of $L_{e}-L_{\mu}-L_{\tau}$ through $\mu_{22,23}$ and vev for $\phi_{2}$ results in finite and calculable corrections to $L_{e}-L_{\mu}-L_{\tau}$ breaking entries of the neutrino mass matrix $M_{\nu}$. In order to evaluate these, it is convenient to work with the original (massless) neutrino flavor basis and treat the mass term $M_{0}^{\nu}$ as an additional interaction. The $H_{a}$ in Fig.1 refer to the mass eigenstates of the charged Higgs fields $H_{a}^{\prime} \equiv\left(\phi_{1}^{+}, \phi_{2}^{+}, \Delta^{+}\right)=O_{a b} H_{b}$.

We have evaluated diagrams of Fig. 1 in the $R_{\xi}$ gauge. Each of the diagrams gives a finite correction to the $L_{e}-L_{\mu}-L_{\tau}$ breaking elements in $M_{0}^{\nu}$ and their sum is gauge independent. One finds,

$$
\begin{aligned}
\left(M^{\nu}\right)_{11}= & \frac{g^{2}}{16 \pi^{2} M_{W}^{2}}\left(M_{0}^{\nu} U^{L} M_{0}^{l} M_{0}^{l \dagger} U^{\dagger L}\right)_{11} \times \\
& \left(1-3 \ln \frac{M_{W}}{M_{3}}-\frac{4 M_{W}^{2} O_{22}}{g^{2}\left\langle\phi_{2}^{0}\right\rangle}\left(\frac{\sqrt{2} O_{32}}{\left\langle\Delta^{0}\right\rangle}-\frac{O_{12}}{2\left\langle\phi_{1}^{0}\right\rangle}\right) \ln \frac{M_{2}}{M_{3}}\right) \\
\left(M^{\nu}\right)_{i j}= & \frac{g^{2}}{32 \pi^{2} M_{W}^{2}}\left(\left(M_{0}^{\nu} U^{L} M_{0}^{l} M_{0}^{l \dagger} U^{\dagger L}\right)_{i j}+\left(M_{0}^{\nu} U^{L} M_{0}^{l} M_{0}^{l \dagger} U^{\dagger L}\right)_{j i}\right) \times \\
& \left(1-3 \ln \frac{M_{W}}{M_{3}}-\frac{4 M_{W}^{2} O_{12}}{g^{2}\left\langle\phi_{1}^{0}\right\rangle}\left(\frac{\sqrt{2} O_{32}}{\left\langle\Delta^{0}\right\rangle}-\frac{O_{22}}{2\left\langle\phi_{2}^{0}\right\rangle}\right) \ln \frac{M_{2}}{M_{3}}\right) .
\end{aligned}
$$

$i, j$ in the above equation take the value 2 and 3 only. $M_{0}^{l}$ and $U^{L}$ are defined in eq.(6). We have repeatedly used the orthogonality of the matrices $U^{L, R}$ and $O$ in arriving at finite result. $M_{2,3}$ refer to the masses of the two physical charged Higgs fields one of which is very heavy, i.e. $M_{3} \sim M_{H}$. Terms cubic in neutrino masses are neglected in writing the above results.

Although the heavy field decouples in the limit $M_{H}$ very large, its residual mixing of order $\frac{M_{W}}{M_{H}} \sim \frac{m_{0}}{M_{W}}$ with the doublet fields influences the radiative masses. This is explicit in the above equations through the presence of the tree level neutrino mass matrix. This has the consequence that the radiatively generated mass terms also display the basic seesaw structure present at the tree level.

The contributions in eq.(1) depend on all three charged lepton masses but the contribution due to tau lepton dominates over the rest unless $U_{31}^{L}$ is enormously suppressed. We shall assume dominance of this contribution. The (logarithmic) contribution of the $W$ diagram is similar in magnitude to the Higgs contributions containing elements of $O$ if the mixing among doublet 
fields $\phi_{1,2}$ is $\mathrm{O}(1)$. Hence for the numerical estimate we shall concentrate on the $\ln \frac{M_{W}}{M_{3}}$ term. The radiatively corrected neutrino mass matrix then has the structure

$$
M^{\nu} \approx m_{0}\left(\begin{array}{ccc}
2 \epsilon s & c & s \\
c & 0 & \epsilon c \\
s & \epsilon c & 2 \epsilon s
\end{array}\right) .
$$

We have implicitly assumed a real $U_{L}$ and $U_{33}^{L} \gg U_{23}^{L}$ in writing the above structure. The parameter $\epsilon$ is defined as

$$
\epsilon \equiv-\frac{3 g^{2} m_{\tau}^{2}}{32 M_{W}^{2} \pi^{2}} \ln \frac{M_{W}}{M_{3}} U_{13}^{L} U_{33}^{L} \sim\left(7 \times 10^{-5}\right) U_{13}^{L} U_{33}^{L}
$$

when $M_{3} \sim 10^{15} \mathrm{GeV}$.

Let us now look at the phenomenological consequences. As already mentioned, $\Delta_{A} \equiv m_{0}^{2} \sim 10^{-2}-10^{-3} \mathrm{eV}^{2}$ follow when Higgs mass $M_{H}$ is in the range $10^{14}-10^{15} \mathrm{GeV}$. The radiatively corrected mass matrix also implies:

$$
\frac{\Delta_{S}}{\Delta_{A}} \sim 8 \epsilon s \sim\left(4 \times 10^{-4}\right) U_{31}^{L} U_{33}^{L} \leq 2 \times 10^{-4}
$$

The mixing among neutrinos is governed by

$$
K_{l} \equiv U_{L}^{\dagger} U_{\nu}
$$

The ratio $\frac{\Delta_{S}}{\Delta_{A}}$ depends upon unknown values of the mixing among charged leptons. The scale required for the vacuum solution follows if the mixing element $U_{31}$ is small. Indeed, $U_{i j} \sim U_{j i} \sim O\left(\frac{m_{i}}{m_{j}}\right)$, for $i<j$ leads to

$$
\Delta_{S} \sim 10^{-7} \Delta_{A} \sim 10^{-9}-10^{-10} \mathrm{eV}^{2}
$$

The leptonic Kobayashi-Maskawa matrix $K$ is also approximately given in this case by $U^{\nu}$ which provides the required bimaximal mixing. Thus model under consideration leads to vacuum solution to the solar neutrino problem for natural value of the relevant parameters.

Unlike the vacuum case, the MSW [10] solution does not follow naturally in the model. To see this, let us concentrate on the approximate result eq.(10). If $U_{33}^{L} U_{31}^{L}$ is less than $\mathrm{O}(1)$ then one does not get a $\Delta_{S}$ in the range required for the MSW to work inside the Sun even when $\Delta_{A}$ is close to its 
upper limit of $10^{-2} \mathrm{eV}^{2}$. Moreover, the charged-lepton mixings being small, the relevant [11] effective mixing angle $\sin ^{2} 2 \theta_{S} \equiv \frac{4 K_{e 1}^{2} K_{e 2}^{2}}{\left(1-K_{e 3}^{2}\right)^{2}}$ is close to 1 in this case, and one gets energy independent suppression already ruled out [12, 13. at the $99 \%$ CL.

On the other hand, if mixing in the charged-lepton sector, specifically $U_{31,33}^{L}$, is large, there is a possibility that the large mixing among the neutrinos can be compensated by the large mixing among the charged leptons. The effective mixing angle in that case can be appreciably less than $45^{\circ}$. Recent global fit to new experimental results does allow large mixing angle solution if one does not include the Superkamioka results on the day-night asymmetry in the fit. However, in that case, the allowed value of $\Delta_{A}$ is even smaller than in the small-angle case. Specifically, the allowed range for large mixing solution is given by 12

$$
0.6<\sin ^{2} 2 \theta_{S}<0.8 \quad ; \quad 8 \times 10^{-5} \mathrm{eV}^{2}<\Delta_{S}<2 \times 10^{-4} \mathrm{eV}^{2} .
$$

It follows that even though proper choice of $U_{31}^{L}$ can lead to the correct $\sin ^{2} 2 \theta_{S}$, eq.(10) cannot lead to the $\Delta_{S}$ in the required range. There is the possibility that the Higgs contribution we have neglected might, for some choice of Higgs and charged-lepton mixing, give rise to $\Delta_{S}$ in the allowed region. However, this would be a marginal case.

The generalization of above results to $S U(5)$ model is straightforward. As an illustration, consider a model with a 15 -plet $\Delta$ and two Higgs $\overline{5}$-plets $\phi_{1,2}$. The $L_{e}-L_{\mu}-L_{\tau}$ symmetry can be replaced by a $U(1)_{H}$ symmetry under which three generations of $\overline{5}$-plet of fermions carry charges $(1,-1,-1)$ respectively while the corresponding 10-plets have opposite $U(1)_{H}$ charges. $\phi_{2}$ carries charge 2 and rest of the fields are taken neutral. In this case down quarks together with the charged lepton have the mass structure given by $M^{l}$ while up-quark masses are given by the following Yukawa couplings:

$$
-\mathcal{L}_{u}=\Gamma_{a i j}^{u} 10_{i} 10_{j} \phi^{* a}
$$

where $a=1,2$ label the two $\overline{5}$-plets of Higgs. The $L_{e}-L_{\mu}-L_{\tau}$ symmetry allows the following Yukawa textures:

$$
\Gamma_{1}^{u} \equiv\left(\begin{array}{ccc}
0 & \beta_{1} & \beta_{2} \\
\beta_{1} & 0 & 0 \\
\beta_{2} & 0 & 0
\end{array}\right) ;
$$




$$
\Gamma_{2}^{u} \equiv\left(\begin{array}{ccc}
0 & 0 & 0 \\
0 & \beta_{22} & \beta_{23} \\
0 & \beta_{23} & \beta_{33}
\end{array}\right)
$$

It follows that the additional $U(1)_{H}$ symmetry does not lead to any prediction in the quark sector but allows a general structure for the quark masses and mixing.

The trilinear terms in (国) are allowed by $S U(5)$ but break the $U(1)_{H}$ softly. All the previous considerations on the tree level as well radiative neutrino masses go through. However there are additional diagrams similar to Fig. 1 contributing to the neutrino masses. These are obtained from above by replacing $W$ boson, charged leptons and colour singlet Higgs by the heavy charge- $1 / 3 X$-bosons, d-quarks and the colour triplet Higgs bosons respectively. The contribution of these is suppressed due to heavy $X$ mass and due to the fact that colour-triplet Higgs have comparable masses. This is to be contrasted with Fig. 1 which contributes large logarithmic factor due to vastly different Higgs masses in the loop, see eq. (7). Thus previous considerations based on the $S U(2) \times U(1)$ model remain valid in this case.

We have discussed here a specific case of the $L_{e}-L_{\mu}-L_{\tau}$ symmetry in view of its phenomenological interest. But the suggested basic mechanism provides a nice scheme to generate pseudo-Dirac structure for neutrino masses in which some symmetry (e.g. $L_{e}-L_{\mu}$ ) leads to Dirac structure and its violation in the charged lepton sector leads to splitting among the degenerate pair radiatively.

Since there have been numerous schemes [14] for radiative neutrino masses, it is appropriate to contrast the present one from the rest. Large class of radiative models [15] use the original mechanisms proposed by Zee [16] and by Babu [17]. The violation of lepton number at tree level gets communicated radiatively to neutrinos in these schemes. Here, neutrinos have lepton number violating but $L_{e}-L_{\mu}-L_{\tau}$ symmetric masses at tree level and breaking of $L_{e}-L_{\mu}-L_{\tau}$ symmetry gets communicated radiatively.

The most noteworthy feature of the present scheme is the dependence of the radiative corrections on the tree-level neutrino and the charged lepton masses. The former is absent in Zee type of models and radiatively generated contribution is controlled only by the charged lepton masses. This feature makes the radiative contribution here quite small and allows one to obtain extremely suppressed solar neutrino scale relevant for the vacuum solution of the solar neutrino problem. 
The conventional radiative models need introduction of additional singly and doubly charged Higgs fields with masses near electroweak scale. Here the role of the charged singlet is played by corresponding field in the triplet which is very heavy. Thus the present scheme does not predict a light exotic charged Higgs. Theoretically, the conventional models are not easily amenable to grand unification in contrast to the present case. The present scheme is similar in spirit to the seesaw model based on $S O(10)$. In spite of the absence of the right-handed neutrino , the model presented here contains seesaw structure for all the neutrino masses and these masses are closely linked to the mass of the charged leptons. This makes the model fairly predictive and leads to a simultaneous solution for the solar and atmospheric anomalies which to date provide the strongest hints to believe that neutrinos are massive.

\section{References}

[1] Y. Fukuda et al., Phys. Lett. B436, 33 (1998) (hep-ex/9805006); Phys. Rev. Lett. 81 (1998) 1158 (hep-ex/9805021), Phys. Rev. Lett. 81 (1998) 1562 (hep-ph/9807003) and S. Hatakeyama et al. Phys. Rev. Lett. 81 (1998) 2016 (hep-ex/9806038).

[2] A. S. Joshipura, hep-ph/9808261

[3] F. Wilczek, hep-ph/9809509.

[4] See e.g. "Massive Neutrinos in Physics and Astrophysics", R. N. Mohapatra and Palash B. Pal, World Scientific (1991).

[5] V. Barger, R. J. N. Philips and K. Whisnant, Phys. Rev. D24, 538 (1981) ; S. L. Glashow and L. M. Krauss, Phys. Lett. B190, 199 (1987); A.Acker, S. Pakvasa and J. Pantaleone, Phys. Rev. D43, 1754 (1991) ; P. I. Krastev and S. Petcov, Phys. Lett. B286, 85 (1992).

[6] R. Barbieri et al. hep-ph/9807235.

[7] Y. Nomura and T. Yanagida, hep-ph/9807325.

[8] Ernest Ma and Utpal Sarkar, Phys. Rev. Lett. 80 (1998) 5716. 
[9] V. Barger, S. Pakvasa, T. J. Weiler and K. Whisnant, hep-ph/9806387.

[10] L. Wolfenstein, Phys. Rev. D17, 2369 (1978) ; S. Mikheyev and A. Yu. Smirnov, Sov. J. Nucl. Physics, 42, 913 (1985).

[11] Q. Y. Liu and A. Yu. Smirnov, hep-ph/9712493.

[12] J. N. Bahcall, P. I. Krastev and A. Yu. Smirnov, hep-ph/9807216.

[13] C. Giunti, hep-ph/9810272.

[14] A review and reference for models with radiatively generated neutrino masses can be found in K. S. Babu and E. Ma, Mod. Phys. Lett, A4 , 1975 (1989).

[15] J. T. Peltoniemi and J. W. F. Valle, Nucl. Phys. B406, 409 (1993); J. T. Peltoniemi, D. Tommasini and J. W. F. Valle, Phys. Lett. B298, 383 (1993); J. T. Peltonemi, A. Yu. Smirnov and J. W. F. Valle, Phys. Lett. B286, 321 (1992); D.O. Caldwell and R.N. Mohapatra, Phys. Rev. D48, 3259 (1993) ; N. Gaur et al, hep-ph/9806272; Ernest Ma, hep-ph/9807386.

[16] A. Zee, Phys. Lett. 93B, 389 (1980).

[17] K. S. Babu, Phys. Lett. B203, 132 (1988). 

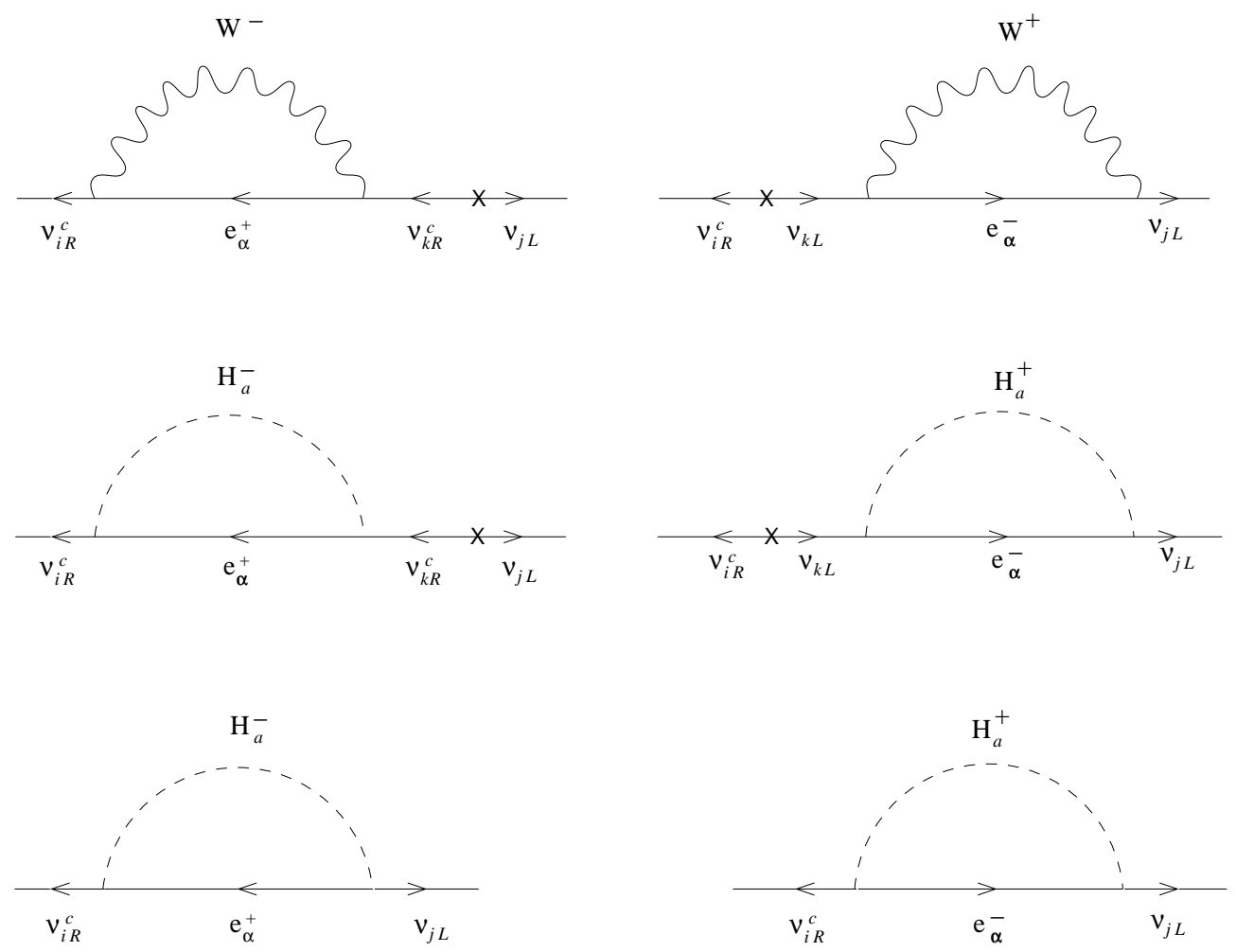

Figure 1: 1-loop diagrams contributing to the $L_{e}-L_{\mu}-L_{\tau}$ breaking entries of the neutrino mass matrix. 